\title{
SURFACE/BULK MICROMACHINED HIGH PERFORMANCE SILICON MICRO-GYROSCOPE
}

\author{
Dong-il (Dan) Cho \\ Department of Mechanical Engineering, University of Califomia, Berkeley \\ Berkeley, CA 94720, USA \\ Sangwoo Lee and Sangjun Park \\ School of Electrical Engineering and Computer Science, Seoul National University \\ Kwanak-gu, Seoul 151-742, Korea
}

\begin{abstract}
A single-crystalline, single-wafer micro-gyroscope is fabricated using the Surface/Bulk Micromachining (SBM) process. The structural thickness of fabricated micro-gyroscope is $40 \mu \mathrm{m}$, and the sacrificial gap is $50 \mu \mathrm{m}$. For electrostatic actuation and capacitive sensing a new electrical isolation method, which uses sandwitched oxide, polysilicon and metal films, is developed. The measured noise equivalent random angle walk of the SBM fabricated gyroscope is $0.0025 \% / \mathrm{sec} / \sqrt{\mathrm{Hz}}$. The output is measured to be linear up to $\pm 20 \% \mathrm{sec}$.
\end{abstract}

\section{INTRODUCTION}

Increasing the structural thickness and using single-crystalline silicon to achieve improved performance for gyroscopes have been an important research topic in recent years. Many process techniques for fabricating High-Aspect Ratio Structures (HARS), such as the SOI [1], epi-poly [2], SOG [3], and SBM [4] processes have been developed. The epi-poly process can have problems of residual stress or stress gradient. The high cost and the residual stress resulting from the bonding process, as well as the footing phenomenon, are the main disadvantages for the SOI and SOG processes.

This paper presents a single-crystalline, single-wafer gyroscope fabricated using the SBM process. A new isolation method for electrostatic actuation and capacitive sensing is also developed in this paper.

\section{EXPERIMENTAL DETAILS}

The SBM technology utilizes standard (111) silicon wafers, and arbitrarily-shaped, released bulk-silicon structures can be fabricated in a single wafer. The SBM process yields very flat and smooth top and bottom surfaces as shown in Fig. 1. It is also free from the footing effects. We have fabricated structures ranging from 2-100 $\mu \mathrm{m}$ in thickness with a sacrificial gap of 2-100 $\mu \mathrm{mm}$. Note that the material properties are transversely isotropic on Si (111) planes, which is very desirable.

Several methods have been reported for electrical isolation of the single-crystalline silicon MEMS [4]-[7]. All of these processes have limitations for application to HARS, which for brevity are not explained in detail.

This paper develops a new oxide/polysilicon/metal triple film isolation method. The detailed process is shown in Fig. 2. In Fig. 2, it is assumed that a silicon microstructure is already fabricated. The isolation process starts with the oxidation of all exposed surfaces (Fig. 2(a)), followed by heavily-doped LPCVD polysilicon deposition (Fig. 2(b)). Since LPCVD polysilicon films have an excellent step coverage, they are deposited on all exposed surfaces. Then, an Al film is sputtered or evaporated (Fig. 2(c)). The polysilicon films at the exposed bottom areas are then anisotropically etched away (Fig. 2(d)), using the top Al layer as the etch mask. Electrical isolation is obtained in this step. Figure 3 shows SEM photographs of an electrode fabricated by the SBM process and this triple film isolation technology.

A gyroscope fabricated using the SBM and triple film isolation method is shown in Fig. 4. The structural thickness is 40 $\mu \mathrm{m}$, and the sacrificial gap is $50 \mu \mathrm{m}$. The overall size is $2.2 \mathrm{~mm} \times 3 \mathrm{~mm}$. The driving and sensing modes of the gyroscope are decoupled, and the resonant frequencies of driving and sensing modes are 4.6 and $5.7 \mathrm{kHz}$, respectively. The total sensing capacitance is $3.12 \mathrm{pF}$.

\section{RESULTS}

Figure 5 shows the results when a $10 \% \mathrm{sec}, 11 \mathrm{~Hz}$ angular rate is applied. The test was performed in a $10 \mathrm{mT}$ Torr vacuum chamber. A 0.8 volt peak-to-peak sinusoidal voltage with 2.5 volt offset was used for driving. The results in Fig. 5 gives a noise equivalent resolution of $0.01 \% \mathrm{sec}$. The measured bandwidth is $16.2 \mathrm{~Hz}$. This gives a noise equivalent random angle walk of $0.0025 \% / \mathrm{sec} / \sqrt{\mathrm{Hz}}$. The output is measured to be linear within $2 \%$ for $\pm 20 \% \mathrm{sec}$ range.

\section{CONCLUSIONS}

In this paper, a single-crystalline micro-gyroscope was fabricated on a single wafer for the first time. The SBM process and the newly developed triple film isolation method were used. The fabricated micro-gyroscope has a noise equivalent random angle walk of $0.0025 \% / \mathrm{sec} / \sqrt{\mathrm{Hz}}$, which is the lowest in any reported silicon gyroscopes to date.

\section{REFERENCES}

1. Diem, M.T. Delaye, F. Michel, S. Renard, and G. Delapoerre, "SOI (SIMOX) as a substrate for surface micromachining of single crystalline silicon sensors and actuators," Tech. Dig. Transducers'93, Yokohama, Japan, June 1993, pp. 233-236.

2. Geiger, B. Folkmer, J. Merz, H. Sandmaier, and W. Lang, "A new silicon rate gyroscope," Proc. IEEE MEMS'98, Heidelberg, Germany, Feb. 1998, pp. 615-620.

3. S. Baek, Y. S. Oh, B. J. Ha, S. D. An, B. H. An, H. Song, and C. M. Song, "A symmetrical z-axis gyroscope with a high aspect ratio using simple and new process," Proc. IEEE MEMS'99, Orlando, FL, Jan. 1999, pp. 612-617.

4. S. Lee, S. Park, and D. Cho, "The surface/bulk micromachining (SBM) process: a new method for fabricating released microelectromechanical systems in single crystal silicon," IEEE/ASME J. Microelctromech. Syst., vol. 8, no. 4, pp. 409-416, Dec. 1999.

5. K. A. Shaw, Z. L. Zhang, and N. C. MacDonald, "SCREAM I: a single mask, single-crystal silicon, reactive ion etching process for 
microelectromechanical structures," Sens. Actuators A, vol. 40, pp. 63-70, 1994.

6. U. Sridhar et. al., "Trench oxide isolated single crystal silicon micromachined accelerometer," Tech. Dig. IEDM'98, San Francisco, CA, Dec. 6-9, 1998, pp. 475-478.

7. S. Lee, S. Park, and D. Cho, "Surface/bulk micromachining (SBM) process and deep trench oxide isolation method for MEMS," Tech. Dig. IEDM'99, Washington, D.C., Dec. 1999, pp. 701-704.

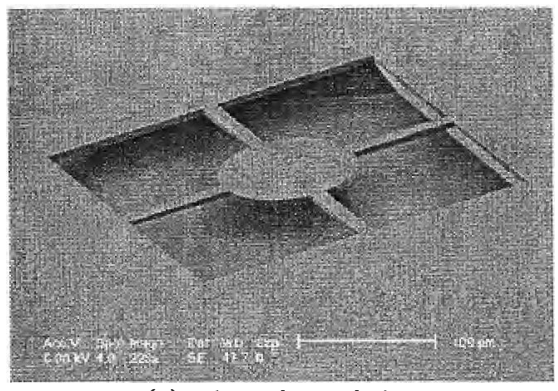

(a) released round plate

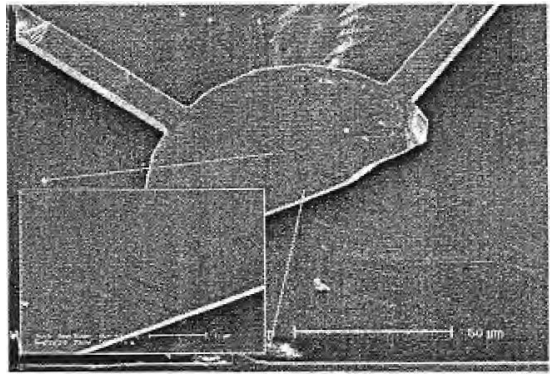

(b) backside of round plate

Figure 1. Examples of fabricated structures [7]

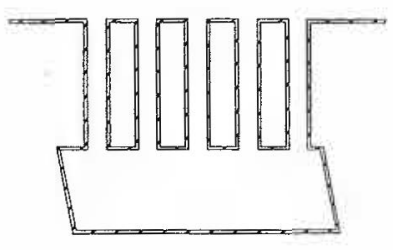

(a) oxidation of released structure

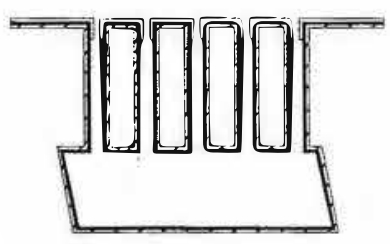

(c) metal deposition

Figure 2. Process flow of developed isolation method.

(b) polysilicon deposition

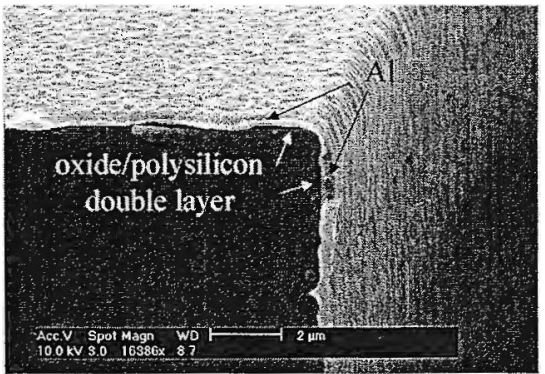

(a) upper part

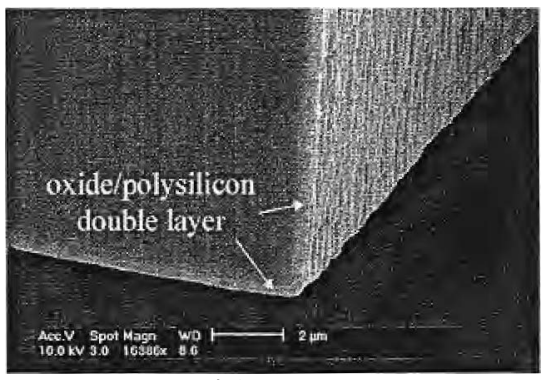

(b) lower part

Figure 3. Electrode processed by the oxide/polysilicon/metal isolation method (40 pus height).

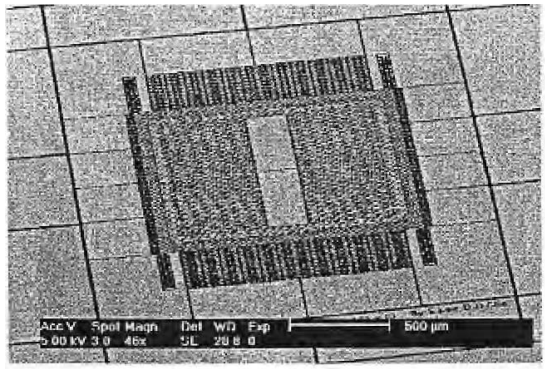

(a) overall view
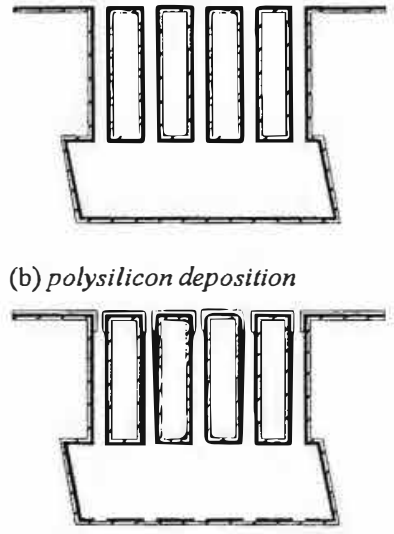

(d) polysilicon etch for electrical isolation (40 um height, 50 an sacrificial gap)

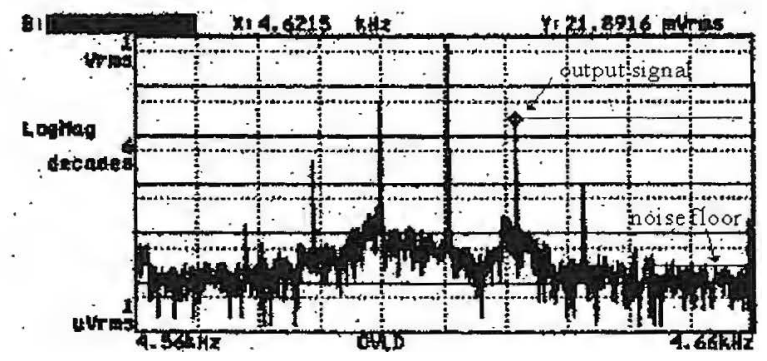

Figure 5. Output Signal of fabricated micro-gyroscope in frequency domain 